\title{
Comportamiento Sexual de los Adolescentes de la Institución Educativa San Juan Bosco - Municipio de Palermo - Huila
}

\section{Sexual Behavior of Teenegers from San Juan Bosco School- Municipality of Palermo - Huila}

Nohora Rojas Losada Psychology Master, Master of Sciense. Universidad Surcolombiana, Facultad de Educación-Dpto. de Psicopedagogía, Neiva. Colombia.

Víctor Javier Vera Cárdenas Psychology Master. Universidad Cooperativa de Colombia. Neiva. Colombia.

\section{Resumen}

El objetivo de la investigación se enfoca a identificar el comportamiento sexual de los estudiantes de la Institución Educativa San Juan Bosco en el Municipio de Palermo-Huila. La metodología empleada responde a un estudio Empírico-Analítico, estudio cuantitativo no experimental, utilizando la técnica descriptiva de análisis. Se aplicó un Muestreo Probabilístico, el cual estuvo conformado por 127 estudiantes de los grados de $6^{\circ}$ a $11^{\circ}$.

Los resultados obtenidos a través de la aplicación de un instrumento validado, informaron sobre las prácticas, conocimientos y opiniones de los adolescentes en torno al comportamiento sexual. El análisis indica como un porcentaje alto de estudiantes (67\%), no tiene claridad sobre lo que realmente es una práctica sexual penetrativa y muy pocos expresan lo que es una práctica no penetrativa. En la descripción de la sexualidad: conocimientos de los estudiantes, se evidencia que cuando se busca información sobre el sexo ellos están interesados en saber en el cómo se desarrollan las relaciones sexuales. La información sobre el aborto es clara; con relación a la genitalidad representa los órganos sexuales y no a las relaciones que se puedan realizar en pareja. Poseen buen conocimiento sobre las zonas erógenas del cuerpo humano que generan placer y tienen claro en que consiste la fecundación, el periodo menstrual, la función de los testículos, las ITS, acoso y abuso sexual, masturbación, aborto, sexo sin afectividad, promiscuidad y madresolterismo. Con referencia a las opiniones de los estudiantes, se obtiene información expresada en un alto porcentaje de estudiantes, que son sus amigos las personas a quienes consultan sobre el sexo.

Palabras claves: Comportamiento sexual, Prácticas, Conocimientos, Opiniones.

\section{Abstract}

This research is aimed at identifying the sexual behavior of teenagers from San Juan Bosco School in the municipality of Palermo, Huila. The methodology implemented consists of an empirical, analytical, quantitative non experimental study that uses a descriptive analysis technique. A probabilistic sampling was applied, which was made up of 127 students from 6 th and 11 th grade levels.

The results obtained from the application of a validated instrument provided information of practices, knowledge and opinions of teenagers regarding their sexual behavior. The analysis indicates that a high percentage (67\%) does not have a clear understanding of what a penetrating sexual practice entails and very few define what this practice entails. Regarding the description of sexuality: students 'knowledge, it is evidenced that they are mostly interested in knowing how sexual relationships are carried out. Information regarding abortion is clear. As for genitality, it does not represent the relationships that couples can carry out, but the sexual organs. They show good knowledge of the erogenous zones of the human body which generate pleasure; they also bear clear understanding of fertilization, the menstrual cycle, the function of testicles, STDs, sexual harassment and abuse, masturbation, abortion, sex without love, promiscuity and single mother circumstance. With reference to students 'opinions, a high percentage of them resort to friends for sex questions.

Keywords: Sexual behavior, practices, knowledge, opinions. 


\section{Justificación}

El estudio presenta una importante trascendencia en la orientación de adolescentes en el tema de la sexualidad. Esto responde a la necesidad exigida desde el Ministerio de Educación y la Secretaría de Educación a nivel del Departamento del Huila, para enfocar concretamente el fortalecimiento de proyectos pedagógicos de educación sexual planteados en el Proyecto Educativo Institucional (PEI- Artículo 73, Ley 115-1994), para la incorporación en la vida cotidiana de los adolescentes el ejercicio de los derechos humanos, sexuales y reproductivos y lograr de esta manera realizar la toma de decisiones que los lleve a vivir una sexualidad sana, plena y responsable para que se enriquezca su proyecto de vida personal y el de los compañeros. Se percibieron aportes fundamentales para la relevancia de la investigación, que contextualizó la situación institucional y que al tenerse en cuenta representan aportes interesantes en el trabajo de orientación.

Además se puede decir que el estudio fundamenta la base para la iniciación de un proceso de reeducación sexual en adolescentes, describir la sexualidad: prácticas, conocimientos y opiniones de los estudiantes entre los 12 y 18 años de la Institución San Juan Bosco en el Municipio de Palermo Huila, que permita la confrontación, clarificación de los conceptos errados, modificación de las actitudes para la construcción de una persona renovada, autónoma, capaz de decidir responsablemente y compartir en la interacción con los demás, sentimientos de afecto, respeto, amor, únicos valores que hace concebir una sociedad donde la tolerancia, la ternura y la libertad no sean un ideal sino una realidad.

Es importante la realización de este estudio en consideración a que la etapa de la adolescencia es la etapa del ser humano en donde se evidencian notablemente los cambios tanto corporales, intelectuales, emocionales y se van estructurando las bases del desarrollo de la personalidad; es una transición de la infancia a la madurez, desde luego influenciada por la cultura, el medio social así como también la historia familiar. Es un ciclo que se extiende de los 10 a los 18 años, período a través del cual se va adquiriendo la madurez psicológica, capacidad reproductiva e independencia económica en la mayoría de los casos. Así mismo, al obtener las informaciones a través del estudio, se considera un buen punto de partida como aporte a la institución para abordar los problemas de los adolescentes, que como acción preventiva, optimizaría las condiciones de riesgos, abusos y explotación sexual que afectan la identidad y el desarrollo de la personalidad del adolescente, como consecuencia de una relativa independencia.

El aporte del estudio se enfoca directamente para que la Institución San Juan Bosco de Palermo Huila, pueda contar con una herramienta de trabajo de orientación a adolescentes y padres de familia para coadyuvar en la formación y en la vivencia adecuada de la vida sexual. Los resultados ofrecidos con base en el estudio, servirá a los directivos de la institución educativa para aunar un mayor interés en reforzar alternativas para generar un bienestar y mejor ambiente, a través de estrategias que fortalezcan las relaciones interpersonales entre los diferentes estamentos académicos (padres, adolescentes y maestros).

Así mismo, representa un aporte a las Ciencias Sociales desde el punto de vista enfocado a utilizar la idea de sexualidad natural, atendiendo a responder los problemas sociales, organizándose frente a las amenazas como el VIH y la congruencia evidente entre normas y prácticas, entre conductas y opiniones, entre acciones y como también el conocimiento y preferencia sexual.

Desde esta dimensión social se abarca igualmente, la relación de los procesos y fenómenos que hacen parte del ser humano como ser fundamentalmente social y político que contribuye a una forma determinada de vivir la sexualidad, es el producto de una construcción social y colectiva, por lo tanto arbitraria, por sus mitos, ritos y otros aspectos.

Desde el punto de vista del tema de estudio, se encuentra una clara justificación puesto que el tema de la sexualidad en el sector educativo tiene una gran importancia y utilidad en investigaciones de orden psicosocial y muy específicamente cuando se intenta analizar la identificación del comportamiento sexual de los adolescentes en la Institución. Por lo tanto, se considera que el presente estudio contribuirá a la realización de planes de orientación en las instancias pertinentes de la institución, en las instancias municipales de salud y en la comunidad en general.

Desde el punto de vista profesional, se adopta una estructura mucho más concreta de ética, valores y normatividad legal que son conformados socialmente para regular el comportamiento sexual y son la base para la organización y planeamiento de la orientación sobre la sexualidad en las instituciones educativas.

\section{El Problema}

Variables como la existencia en el municipio un alto índice de niños, adolescentes y jóvenes que aplican en el sistema educativo, son motivo de análisis en el estudio de la problemática planteada. Se aprecia con relación a la cobertura de estudiantes en el sistema educativo, el SIMAT - Anexo 6A de 01-06-13, que se presenta en la zona urbana a nivel departamental una inscripción de 69.436 estudiantes y en la zona rural: 78897 estudiantes. Palermo en términos porcentuales presenta una participación del 4\% para la zona urbana y el $3.5 \%$ para la zona rural. A nivel del municipio se identifican 5618 estudiantes matriculados, de los cuales el $51 \%$ pertenece a la zona urbana y el $49 \%$ a la zona rural respectivamente. Para el caso del estudio se analizó el comportamiento de matriculados en los grados $6^{\circ}, 7^{\circ}, 8^{\circ}, 9^{\circ}, 10^{\circ}$ y $11^{\circ}$, con una participación del $33 \%$ y la matrícula de la Media el 10\% Correspondiente especificamente a estudiantes adolescentes matriculados en el sector oficial (SIMAT, 06-13). 
La Institución Educativa San Juan Bosco, fue la institución seleccionada para el estudio, como centro oficial y convergente de una gran población estudiantil en el municipio de Palermo. Fundado por el sacerdote Teódulo Monje en el año de 1996, con el acuerdo del señor alcalde Delfín Cubillos Conde.

Se observa a nivel del municipio el embarazo temprano o adolescente; el cual no se planea y sucede en parejas económicamente dependiente de otras personas, que pueden ser sus padres o sustitutos, con características tipificadas con relaciones no estables y que en la mayoría de los casos compromete el proceso de desarrollo representado en: Estudio, empleo o proyectos de vida que se truncan, por la falta de madurez tanto física como psicológica. En el Huila la gestación temprana en los municipios, presenta un comportamiento que está por encima de la meta de reducción esperada por debajo del $15 \%$. Palermo presenta el 39\% de adolescentes gestantes de la población y si se analiza por edades, entre los $10 \mathrm{y}$ los 14 años se presenta el $2.8 \%$ de la población adolescentes. Los embarazos en menores de 14 años se considera delito penal, presentándose para el año 2012, 366 casos a nivel departamental. (Informe de Gestión para la garantía de los derechos de la infancia, adolescencia y juventud - Dpto. del Huila, Julio 20112012).

La infección por el Virus de Inmunodeficiencia Humana (VIH) y el síndrome de Inmunodeficiencia Adquirida (SIDA), han generado implicaciones económicas altas al sistema general de seguridad social en salud. Obviamente son calamitosos los efectos sobre la calidad de vida de las personas que viven con el virus y sus familias, además de la repercusión de orden laboral. En el año 2012 se han reportado 128 casos de VIH/SIDA y muerte por SIDA en el Departamento del Huila. La prevalencia de la epidemia VIH es de $0.25 \%$ en la población entre 15 y 49 años.

Según los informes sobre VIH/SIDA, departamento del Huila, 1988-2012, observados en el Boletín epidemiológico Departamento del Huila, (2012) el "98\% de la población en el departamento del Huila se infecta por transmisión sexual, y a su vez se tiene que el $70 \%$ es de tipo heterosexual, $20 \%$ homosexual y $7 \%$ bisexual, y un $2 \%$ de orientación sexual desconocida; se reporta un caso por uso de drogas IV". Ningún caso reportado por transmisión perinatal, transfusión sanguínea o accidente laboral en el 2012. (p. 5)

Palermo no figura para la fecha anterior, entre los municipios afectados por esta epidemia en las estadísticas oficiales, pero se reporta por vía oral, la presencia de 9 casos en el año 2012, en la población entre 14 a 18 años, que se han trasladado a otras ciudades por razones de tipo personal y social. Sin embargo, para el año 2007, la Secretaría de Salud del Municipio de Palermo reportó a la Secretaría de Salud Departamental 22 casos de personas con VIH/SIDA, con una incidencia del $68 \%$ en hombres adultos. Para ese momento la tasa de prevalencia llegó al 0.7 por 1000 habitantes.

La aparición de sífilis congénita, como una ITS que se descubre en el primer trimestre de la gestante, su agente causal es el Treponema pallidum y su transmisión se da de manera sexual y transplacentaria. En el Departamento del Huila en el 2012 se detectaron 122 casos de sífilis gestacional, que indicaron una razón de prevalencia de sífilis gestacional de 5.5 casos por 1.000 nacidos vivos. Palermo presenta $0.4 \%$ de la población gestante adolescente con sífilis gestacional. Obviamente es un porcentaje bajo pero debe ser motivo de análisis el área de procedencia y la sobrepoblación rural y urbana que se está presentando por el desplazamiento forzoso. A nivel del Departamento del Huila, se han presentado 88 casos en cabecera municipal y 23 casos en la zona rural.

En lo referente al abuso sexual, considerado un fenómeno negativo, se ha reportado en el 2008, 18 casos de abuso sexual; se presentó el caso de una menor de 4 años que representa el 5.5\% del total de los casos; 5 casos de niñas entre 5 a 9 años, equivalente al $27.7 \%$ y un $66.6 \%$ de los casos corresponde a 12 adolescentes de género femenino. Teniendo en cuenta la población infantil en el municipio, la incidencia es la siguiente: en menores de 4 años la incidencia es de 0.66 por 1.000 habitantes, en menores de 5 a 9 años es de 3.4 por 1000 habitantes y de 10 a 17 años de edad se presenta una incidencia de 0.43 por 1000 habitantes. Como se puede apreciar, aunque en la edad adolescente se presentó el mayor número de casos, por tasa de incidencia son los menores de 5 a 9 años quienes estas en mayor riesgo de ser víctimas.

Pandi, Agencia Periodismo Amigo de los Derechos de la Infancia (2006). Tomado el 19 de Agosto de 2013 de http://www.semana.com/on-line/articulo/aumentaexplotacion-abuso-sexual-ninos-colombia/86676-3. Revista Semana, sección La Nación, 2007), muestra en cifras los últimos datos sobre el abuso infantil en Colombia, en la siguiente forma:

- "Durante 2006 el abuso sexual infantil en Colombia aumentó en un $6 \%$ respecto al año anterior. El rango más alto se encuentra en niños y niñas entre 5 y 14 años de edad.

- Bogotá y Antioquia son identificadas como las zonas en donde más se cometen abusos.

- Se denunciaron 14.840 casos ante Medicina Legal en el último año.

- El 86\% de las víctimas de abuso sexual que se presentan en Medicina Legal son menores de edad.

- 12.247 niñas y 2.593 niños fueron abusados sexualmente durante el año 2012.

3 de cada 100 menores asistieron a Medicina Legal por abuso sexual".

El silencio es la cifra más importante pues se estima que el $70 \%$ de los casos no es denunciado por temor, conflictos sociales o coerción.

- Existen 2406 casos reportados indirectamente por institutos médicos o servicios sociales obligatorios. 
- Cada 14 minutos se presume que se presenta un caso de abuso sexual contra un menor de edad en Colombia, que es denunciado. Se cree que la cifra de casos no denunciados es mucho mayor". Revista Semana, sección La Nación, 2007.

Así mismo, la presencia de personas que han sido víctimas en Colombia del conflicto armado y la delincuencia común, ha afectado nuestro departamento y muy particularmente al municipio de Palermo. Las personas menores de 18 años en condición de desplazamiento en el departamento del Huila, registran un crecimiento del $3.2 \%$ en el año de 2010 con relación al 2007. Según el SIPOD-Acción Social (2011), "del total de esta población para ese año correspondía al 47.2\% menores de edad y en el año 2010, ascendió a $50.4 \% "$. (Informe de gestión para la garantía de los derechos de la infancia, adolescencia y juventud Dpto. del Huila, Neiva Julio 2011).

Es una grave situación que se ve reflejada en la invasión de espacios en determinados barrios del casco urbano de Palermo, habitados en su mayoría por personas que han sido desplazadas de su lugar de origen teniendo que dejar lo que hasta su momento habían conseguido, sus casas, fincas, cultivos, animales, electrodomésticos, el estudio de sus hijos, entre otros. Pero lo que trae como efecto colateral, el asentamiento de esta nueva población, es la desconfiguración los procesos sociales, que afectan el comportamiento de los niños y adolescentes en el municipio.

La tranquilidad de la región se ha tornado difícil puesto que esta serie de variables mantiene en alerta tanto a las familias, los educadores como a las instituciones educativas, lo cual enfoca la urgencia de tratar los efectos que se reflejan en los comportamientos de los adolescentes. Por ejemplo, el tema de la sexualidad es una seria problemática social, que se ha venido observando a través del tiempo; presentándose en los adolescentes conductas que no corresponden a su etapa de desarrollo dentro del proceso evolutivo, puesto que actúan sin responsabilidad y madurez, posiblemente por la influencia de su entorno. Evidentemente este tipo de actuaciones se refleja en los riesgos a que se exponen, abusos y en algunas oportunidades a explotación sexual.

Por estas razones y aspectos planteados anteriormente surge la iniciativa de realizar un estudio a través del cual se pueda determinar ¿Cuál es el comportamiento sexual en los adolescentes entre los 12 y 18 años de la Institución Educativa San Juan Bosco del municipio de Palermo-Huila?

\section{Objetivo General}

Describir el comportamiento sexual de los estudiantes de la Institución Educativa San Juan Bosco en el Municipio de Palermo-Huila.

\section{Objetivos Específicos}

Identificar las prácticas sexuales de los estudiantes entre los 12 y 18 años de la Institución Educativa San Juan Bosco en el Municipio de Palermo Huila.

Identificar los conocimientos sobre la sexualidad en los estudiantes entre los 12 a 18 años de la Institución Educativa San Juan Bosco en el Municipio de Palermo Huila.

Identificar las opiniones respecto a la sexualidad de los estudiantes entre los 12 a 18 años de la Institución Educativa San Juan Bosco en el Municipio de Palermo Huila.

\section{Metodología}

\section{Tipo de Estudio}

Se realizó un estudio cuantitativo no experimental, aplicando como técnica de análisis el descriptivo, con la finalidad de identificar el comportamiento sexual de los estudiantes de la Institución Educativa San Juan Bosco en el Municipio de Palermo-Huila. Se adoptó este estudio porque se basa directamente en el paradigma explicativo, a través del cual se explicaron los fenómenos de estudio propuesto. Los estudios de investigación cuantitativa tienen como objetivo adquirir conocimientos fundamentales a través de un modelo adecuado que permita conocer la realidad en forma imparcial ya que recogen y analizan los datos a través de los conceptos y variables (Briones, 1996).

\section{Población y Muestra}

La población de estudio estuvo conformada por los alumnos de grado $6^{\circ}$ a grado $11^{\circ}$, de la Institución Educativa San Juan Bosco de Palermo, constituido por 191 estudiantes entre 12 y 18 años de ambos sexos, del nivel medio y básico de la mencionada institución a saber: 30 estudiantes del grado sexto, 35 del grado séptimo, 32 del grado octavo, 32 de noveno, 32 del grado décimo y 30 del grado once.

Para determinar la muestra se utilizó un muestreo probabilístico, el cual estuvo conformado por 128 sujetos, niños y niñas de los diferentes grados, a quienes se les aplicó el instrumento y su conformación se hizo al azar. Para el caso, se aplicó la muestra estratificada proporcional: Las unidades se dividieron en estratos, eligiendo al azar dentro de cada estrato, quedando distribuida la muestra de la siguiente manera: 21 estudiantes del grado sexto, 23 del grado séptimo, 21 del grado octavo, 21 de noveno, 21 del grado décimo y 21 del grado once.

\section{Instrumento}

El instrumento fue diseñado por la investigadora y revisado por el asesor Psicólogo Víctor Javier Vera; contiene 26 ítems. Además, este instrumento contó 
con la revisión del Doctor Guillermo Sánchez Amaya, Sociólogo investigador adscrito al Departamento de Psicopedagogía de la USCO. Para efecto de validar el contenido de la misma fue revisado por psicólogos expertos para precisar los ítems que finalmente constituyeron la encuesta. En la realización de este proceso evaluativo del instrumento se suministró a los Jueces asignados, la tabla de variables con los respectivos ítems correspondientes a cada pregunta en el instrumento, los cuales con base en los parámetros: suficiencia, coherencia, relevancia y claridad, emitieron su concepto evaluativo. En el procedimiento se presenta una descripción en torno a cómo se llegó a la construcción del instrumento tipo encuesta.

Para efectos de validez, se utilizó la validez de contenido en la cual cada reactivo debe ser juzgado con base en su relevancia presumida respecto a la propiedad que se va a medir. Por consiguiente lo que se pretende es evaluar la representatividad muestral del contenido del instrumento. En cuanto a la confiabilidad del instrumento, no se empleó ningún tipo de método estadístico debido a que la encuesta no fue diseñada con fines de estandarizar los resultados. Se realizó una prueba piloto, tomando una muestra representativa de 25 estudiantes (30\% de la muestra seleccionada: 124 estudiantes), de $6^{\circ}$ grado a $11^{\circ}$. No hubo ningún inconveniente de interpretación literal.

\section{Descripción del instrumento}

El instrumento diseñado tipo encuesta, presenta solamente una versión para aplicar a niños y adolescentes de grado $6^{\circ}$ y grado $11^{\circ}$.

La encuesta quedó conformada por 26 ítems (Anexo No. 3), tomando como base las sugerencias realizadas por los expertos jueces asignados y compuesta por:

I. Información general

II. Prácticas sobre la sexualidad

III. Conocimiento sobre la sexualidad

IV. Opiniones sobre la sexualidad

\section{Discusión-Resultados}

Con base en la aplicación del instrumento diseñado para la investigación y los datos obtenidos a través de su realización en 127 adolescentes, se infiere que tanto el área de salud como la de educación enfrenta con la población adolescente una problemática recurrente, frente a las actitudes demostradas en prácticas, conocimientos y opiniones sobre la sexualidad.

Una educación integral de la sexualidad implica que mujeres y varones conozcan e identifiquen los aspectos anatomofisiológicos, psicosociales y culturales de la sexualidad que les permita asumir el compromiso de ejercerla de manera responsable. De ahí la importancia de conocer las prácticas, conocimientos y opiniones que esta franja de la población manifiesta ya que marca parámetros de análisis sobre sus conductas sexuales.

El nivel educativo de los encuestados se ubica entre $6^{\circ}$ y $11^{\circ}$ grado; y como lo expresa $\mathrm{El}$ director ejecutivo de UNFPA, Doctor Babatunde Osotimehin, al celebrar el día mundial de la población (10-VII-2013), a los adolescentes se les debe ofrecer una educación integral sobre la sexualidad apropiada a sus edades con el objetivo de proteger su salud a través de la vida. Desde luego se hace referencia al hecho de identificar en su proceso de formación las variables que se han tomado para el desarrollo del trabajo, como aspectos que lo enfocan al manejo adecuado de su sexualidad.

En las (os) adolescentes participantes, se encontró un alto porcentaje de ellos que consideran como práctica sexual penetrativa las relaciones interrumpidas. Sin embargo en una proporción más baja otros estudiantes piensan que las prácticas sexuales no penetrativas son aquellas en donde únicamente se hace un contacto personal, seguido de un buen número de estudiantes que manifiestas no tener ninguna información sobre el tema. Pero al igual llamadas telefónicas eróticas, sexo virtual a través de internet, fetichismo, pornografía son identificadas por una minoría como una práctica sexual no penetrativa, la cual se puede asociar a la masturbación.

De acuerdo a lo expresado por Ortiz (2011), con relación a las prácticas sexuales penetrativas, existe una proporción alta sobre la claridad conceptual de los estudiantes en lo referente a las prácticas penetrativas. Penetración puede referirse a la penetración sexual o coito. Así mismo se constata que de acuerdo al nivel de respuesta no es muy claro definir las prácticas sexuales no penetrativas; nos son explícitos, sin embargo las consideran no riesgosas, debido a la no penetración e intercambio de secreciones corporales que contienen, por ejemplo, el VIH u otro agente de ITS. Tal y como lo expresa Cubillos (2011) en sus escritos sobre Orientación a Parejas, "el sexo en una necesidad biológica que debe ser sanamente compartida, llevada con responsabilidad y con el fin de reproducción y por placer. Las prácticas sexuales son todas aquellas manifestaciones de contacto físico que por amor, afecto, gusto o placer un ser humano puede realizar con su cuerpo en contacto con sí mismo o con el cuerpo de otro ser humano". (p. 45)

Los resultados del estudio en cuanto al conocimiento sobre el sexo, evidencian que cuando se busca información sobre el sexo lo que manifiestan los estudiantes, se enfoca al interés sobre cómo se desarrollan las relaciones sexuales y representa su mayor expectativa; sin embargo si se realiza con morbo, no es tanto su interés en consideración a que la experiencia no les ha proporcionado aún esta opción que les permita emitir juicio alguno.

Por otra parte, al utilizar el término sexualidad su conocimiento está referido a la información acerca de cómo tener relaciones sexuales. El cuanto a la genitali- 
dad, el 33.9\% de los estudiantes, se ubica al conocimiento de los órganos genitales y un porcentaje inferior considera que genitalidad es acoplamiento coital. Así mismo, cuando se habla de las zonas erógenas en el cuerpo humano se expresa, que son las zonas de mayor sensibilidad erótica en el cuerpo humano que producen placer y es un alto número de estudiantes que presenta este conocimiento. La fecundación es conocida por los estudiantes, como la unión de células sexuales para formar una célula, huevo o cigoto, aunque en porcentaje menor conoce la fecundación como la concepción de un nuevo ser y la menstruación como el flujo de sangre que se presenta en la mujer cada 28 días. Para el grupo de estudio, los testículos presentan como función, la de ser responsables de la producción de espermatozoides.

Referente a los métodos de planificación familiar, los estudiantes han manifestado tener conocimiento sobre las inyecciones, pastillas y el condón. Sobre la vasectomía en el hombre, ligadura de trompas en la mujer, los anovulatorios: orales como la píldora, inyectables en ampollas mensuales o trimestrales, métodos de barreras: los espermicidas y los condones y los dispositivos intrauterinos: $\mathrm{T}$ de cobre, y los dispositivos subdérmicos: Jadel, norplan, no tienen conocimiento.

Por otra parte es bien claro para la mayoría de estudiantes en que consiste el aborto, ya que expresan que es la interrupción del embarazo. Sobre las infecciones de transmisión sexual (ITS), así mismo tienen conocimiento sobre el VIH/SIDA - Sífilis y en menores proporciones aducen que la gonorreaenfermedades pélvicas inflamatorias, el herpes genital y la hepatitis B. La forma de adquirirlas se hace a través de relaciones sexuales con alguien que esté enfermo y no se haya protegido es el conocimiento manifestado por los actores del estudio. El conocimiento sobre acoso sexual expresado por los estudiantes, lo entienden como caricias o agarres no consentidos, hacer daño, ofender o intimidar a otra persona; en tanto que el abuso sexual son actitudes y comportamientos que realiza una persona sobre otra, sin su consentimiento o conocimiento.

Llama la atención los datos suministrados por el grupo de estudio, sobre los conocimientos, que si bien son ciertas las puntuaciones altas a algunas respuestas, existe el convencimiento sobre la necesidad de una mayor información, tal como expresa la por la OEA "Comisión Interamericana de Derechos Humanos (CDH)" Informe Anual 2011, en el estudio sobre Acceso a la información en materia reproductiva desde una perspectiva de Derechos Humanos:

El derecho al acceso a la información es especialmente relevante en el ámbito de la salud y específicamente en el área de la sexualidad y la reproducción ya que contribuye a que las personas estén en condiciones de tomar decisiones libres y fundamentadas respecto de aspectos íntimos de su personalidad. Bajo el sistema interamericano, el acceso a la información en materia sexual y reproductiva involucra una serie de derechos como el derecho a la libertad de expresión, a la integridad personal, a la protección a la familia, a la vida privada y a vivir libres de violencia $y$ discriminación (p. 1).

Así mismo, la conveniencia de la claridad sobre el tema de estudio, es particularmente importante, de tal manera que existe un saber fundamental cuando Cubillos E. (2010), habla sobre la sexualidad, como un sistema de la vida humana y como tal está constituido por unas características que la identifican y que interactúan entre sí y con otros sistemas como el biológico, psicológico y social. Hace referencia a: "el erotismo o aquella capacidad de sentir el placer a través de respuestas sexuales, la afectividad, la reproducción y el sexo genético y físico" (p. 39).

Importante identificar las opiniones que se tienen sobre la sexualidad de acuerdo a la información que cada estudiante asume sobre el tema. Ellos consideran que la sexualidad incluye la parte física, reproductiva y la erótica, la primera relación sexual significará una huella permanente en los futuros encuentros sexuales con una pareja, es el concepto de pocos estudiantes. Consultado el grupo de estudio sobre a quienes se dirige para hacer consultas sobre la sexualidad, expresan que no consultan con nadie y otro grupo considerable acude a los amigos. Ortiz (2010), manifiesta que siempre se ha considerado hablar de sexualidad como un tema tabú, de ahí el interés que los adolescentes presentan de estar especialmente con sus amigos, a través de los cuales adquieren una serie de informaciones, en la mayoría de los casos tergiversada. Se evidencia un tema vedado que no incursiona públicamente (p.10).

Desde luego como lo expresa Ortiz (2010), el medio que rodea al adolescente influye fundamentalmente en su comportamiento, como por ejemplo la ausencia paterna, familia en descomposición, promiscuidad sexual observada de personas en su medio, la carencia de valores y actitudes, inexistencia de la conciencia de la maternidad y/o paternidad, sino que se llega a ella por accidente.

Caricote (2008), en su investigación sobre la influencia de los Padres en la educación sexual de los adolescentes presenta resultados relacionados con:

"..... los padres de familia reflexionaran sobre los conocimientos, actitudes y prácticas sexuales con el ánimo de desarrollar una orientación más puntual, dado que "el aprendizaje de la sexualidad es un hecho propio de la vida cotidiana y a través de la familia, el/la niño/a y el/la adolescente reciben permanentemente mensajes tácitos o explícitos, verbales, no verbales a través de los cuales van teniendo una actitud negativa o positiva inconsciente frente a su cuerpo, su sexualidad y la del otro". (pp. 79-87).

Sobre en qué consiste la masturbación, los encuestados, comenta su opinión referida al acto de 
proveerse placer sexual a través de la estimulación de los órganos genitales y en el hecho de obtener placer sexual a través de la estimulación, una mínima parte de los estudiantes opina en esta forma. Ortiz (2011), comenta que se debe adoptar un cambio de actitud con relación al tema de la masturbación. De hecho se ha considerado por expertos que se puede aliviar la tensión sexual y además es una forma de conocimiento de su propio cuerpo, (p. 28).

La opinión de los estudiantes con relación al aborto, se orienta a expresar que se trata de un crimen, delito abominable y una nefasta solución para la irresponsabilidad de los actos sexuales sin prevención; además expresan los estudiantes que es la destrucción de un embrión o feto, manipulado en el seno materno con el propósito de destruirlo. El embarazo no deseado es uno de los principales problemas que presentan las jóvenes lo cual las conduce al aborto inducido o provocado. El hecho de no poder acceder a los servicios en forma oportuna repercute en el avance del embarazo y es ahí en donde radica la complicación en términos de salud. En el Plan local de salud reproductiva del municipio de Palermo- Huila, (Secretaria de Salud Municipio de Palermo, 2008-2011. Disponible en dirección electrónica: http://www.palermo-huila.gov. co/apc-aa-files/396562383863343634363739613430 33/PLAN_LOCAL_DE_SSR_4.pdf), en el punto sobre el fortalecimiento de la gestión institucional, las acciones propuestas se orientan a: fortalecer la capacidad operativa, técnica y de gestión de las entidades responsables de la prestación de los servicios, diseñar modelos de atención para garantizar accesibilidad a métodos de planificación familiar a oblaciones vulnerables, promover cambios normativos con criterios de costo efectividad, los métodos de planificación familiar y constituir los mecanismos competentes para la adquisición de los medicamentos anticonceptivos (p. 30-31).

En el estudio, así mismo se determina por parte de los estudiantes respecto al sexo sin afectividad opinan que se presenta en situaciones cuando se ocurre abuso sexual, en tanto que unos pocos estudiantes opinan que es la relación sexual que se lleva a cabo cuando se realiza por simple curiosidad.

Se pudo inferir a partir de las respuestas presentadas por los estudiantes a través del presente estudio, que cuando el sexo no se realiza con una sola pareja sino que se realiza con varias personas, este tipo de relaciones son realizadas por deseo y placer. Así mismo, opinaron que este tipo de relaciones se pueden cumplir por trabajo sexual o prostitución o debido a relaciones que se presentan por problemas psicológicos, representada esta opinión en respuestas en una mínima proporción de encuestados. De acuerdo a lo estudiado por Mojica (2011), cuando no se tiene una pareja estable, los riesgos ante las ITS son mayores y puede tender a la falta de seguridad, la insatisfacción sexual que lo lleva a tener sexo indiferentemente, repercutiendo en un problema psicológico de mayores proporciones (p. 87).
La opinión sobre madre soltera por parte de los estudiantes se enfoca a dejar ver cómo la madre afronta la doble carga doméstica y laboral en condiciones desventajosas, debido a la inminente responsabilidad en torno a ofrecer educación, alimento, protección, la formación en valores y afecto para el desarrollo holístico de un nuevo ser humano. De otra parte opinan que para la mujer es una limitación o bloqueo a las oportunidades que se presenten en la vida. Franco (2010), explica que es necesario abordarlo desde la perspectiva cultural en donde ocurre la vida habitual de la adolescente, para desde ese marco poder analizar la problemática y el cómo puede llegar a asumir su nueva condición. La mayoría de estas mujeres son personas carentes de educación y económicamente desprovistos de recursos (p. 14). En cuanto a la influencia en la vida de un adolescente padre soltero, son bien claros en su opinión, puesto que como padre adolecente debe tomar decisiones tan importantes como casarse, convivir con su pareja y adquiere responsabilidades no en el tiempo apropiado sino durante el proceso de desarrollo y formación personal.

Cuando se habla de acoso sexual, opina que es una situación que afecta la dignidad humana por actos contrarios a su voluntad, vulneración de sus derechos como el desarrollo de sus capacidades y en menor proporción opinan que en la incapacidad personal para evitarlo. Y con respecto al abuso sexual, opinan que es un acto agresivo, denigrante que puede traumatizar psicológicamente a la mujer y a la familia. Además, enfocan su opinión a considerar que se atenta contra la libertad del ser humano. El acto de violación es un acto de agresividad verbal, física y psicológica es su opinión y es una conducta irresponsable y agresiva de los adultos que coloca en riesgo la vida de los Adolescentes. Mojica (2011) en su libro: Deformaciones de la Sexualidad, comenta sobre el tema y se infiere: "todo acoso es ofensivo y genera estrés emocional en las personas afectadas sean hombres o mujeres" (p. 67).

A través de la investigación, conformación de la conciencia en el adolescente sobre la sexualidad, Carrasco (2007), y desde la comparación teórica se evidencia, por ejemplo, en los resultados la confirmación de la sexualidad del adolescente como un fenómeno pluridimensional asociado a las dimensiones biológicas, psicosociales, conductuales, morales y culturales, las cuales se deben orientar integradamente. Estas dimensiones permiten al ser humano vivir y comprender el medio en donde nos movemos y ante todo lo que nos rodea. La identificación y la formación de la conciencia sexual ya sea hombre o mujer, se basan en vivencias que experimenta el cuerpo humano y se enfocan estas experiencias a la formación de la personalidad. Además expresa Carraco (2007): "La sexualidad no sólo se refiere a la reproducción o a las relaciones coitales, sino aún más, a los sentimientos, pensamientos, actitudes y comportamientos cotidianos de la vida. o bien, la sexualidad puede ser definida como la 
expresión psicológica y social de los individuos, en una cultura y en un momento determinado, por lo tanto, el estudio de la sexualidad debe realizarse desde diferentes puntos de vista; a las explicaciones biológicas deben agregarse, con toda la importancia que merecen, las que estudian el comportamiento humano en general, lo psicológico y lo sociocultural (p. 2).

El director ejecutivo de UNFPA, doctor Babatunde Osotimehin (2013), ha manifestado la necesidad atencional que se debe dar a los adolescentes en su salud sexual:

"Los servicios de salud reproductiva de buena calidad deben también estar fácilmente disponibles para que los adolescentes tomen decisiones informadas y sean saludables. A nivel local, las comunidades deben ofrecer la infraestructura para entregar cuidado de la salud reproductiva adaptado y sensible a los jóvenes" (p. única).

\section{Conclusiones}

De acuerdo al objetivo propuesto, respecto a la conceptualización sobre la sexualidad: prácticas, en los estudiantes entre los 12 y 18 años de la Institución Educativa San Juan Bosco en el Municipio de Palermo Huila, no tienen claridad sobre lo que realmente es una práctica sexual penetrativa y muy pocos expresan lo que es una práctica no penetrativa asertivamente.

Describir la sexualidad: conocimientos de los estudiantes entre los 12 a 18 años, se evidencia que cuando se busca información sobre el sexo están interesados en saber en el cómo se desarrollan las relaciones sexuales, en tanto que el término sexualidad se enfoca a cómo tener relaciones de tipo sexual. La información sobre el aborto es de buen manejo conceptual y la genitalidad es para estos adolescentes los órganos sexuales y no a las relaciones que se puedan realizar en pareja. Sin embargo, están bien informados sobre las zonas erógenas del cuerpo humano que generan placer y así mismo tienen claro en que consiste la fecundación, el periodo menstrual y la función de los testículos.

No existe un conocimiento avanzado sobre los métodos de planificación existentes, es un tema bastante limitado que no favorece la actitud en la toma de decisiones frente a la sexualidad. Igualmente la información sobre las ITS es pobre, pero su conocimiento no está desenfocado al expresar que se adquieren por relaciones sexuales. Así como se ha expresado en los antecedentes teóricos del estudio, la Organización Mundial de la salud (OMS), es necesario que se les brinde todo tipo de información para la prevención y la salud sexual del adolescente. Con relación al abuso sexual y acoso sexual, son dos expresiones que tienden a confundir, dado el bajo porcentaje de la muestra en torno a la definición concreta de cada una.

Describir la sexualidad: opiniones de los estudiantes entre los 12 a 18 años, se obtiene información expresa- da en un alto porcentaje de estudiantes, que son sus amigos las personas a quienes consultan sobre el sexo. Opinan así mismo, que la sexualidad incluye la parte física, reproductiva y la erótica y que la primera relación sexual significará una huella permanente en los futuros encuentros sexuales con una pareja. Sobre la masturbación son muy pocos los estudiantes que están enfocados para opinar correctamente sobre este tipo de comportamientos. La opinión para algunos estudiantes, sobre el aborto no es concreta, pero se aprecia en el colectivo de la muestra, desconocimiento sobre el tema. De otra parte el sexo sin afectividad, es una situación a la que se llega por abuso sexual.

$\mathrm{El}$ acto de realizar el sexo sin pareja definida, opinan que es una situación que se aboca únicamente por deseo, placer o relaciones por trabajo sexual. Opinan que ser madre soltera-padre soltero, son circunstancias que llevan a asumir responsabilidades prematuras: abandono del sistema escolar y vinculación a la fuerza laborar, bloqueo de oportunidades en su proyecto de vida, entre otras. Siendo en estos casos la mayor afrontación para la mujer, quien a tan temprana edad de adolescente debe responder por la manutención y crianza de su hijo. La opinión sobre acoso sexual-abuso sexual, el acto de violación no es clara para la mayoría de los estudiantes.

Otro hallazgo significativo en la presente investigación, corresponde a la necesidad de una buena disposición de los servicios de salud reproductiva de calidad disponible a los adolescentes para la toma de decisiones informadas, saludables y le corresponde a los gobiernos locales proveer de programas de orientación tanto a los adolescentes como a los jóvenes. Queda claro a partir de estos resultados, que es necesario desarrollar estrategias de prevención en la comunidad.

De igual forma y en el sentido de la conclusión anterior, se evidencia un interrogante relacionado con el tipo de conductas-respuesta, expuestas por los estudiante de la Institución Educativa San Juan Bosco, con relación a las variables de estudio que conduce a pensar que lo adquirido en el grupo familiar es la base del desarrollo personal-sexual, enfocado desde las prácticas, conocimientos y opiniones que poseen sobre la sexualidad y la participación en la formación de estas conductas a través de orientaciones en las instituciones, como complemento de lo adquirido en el seno familiar. Desde luego se infiere que la escuela no es la única responsable de la educación sexual, es un tema que se forma en la persona desde el ámbito académico a partir de cada área del conocimiento a través de un desarrollo transversalizado.

\section{Recomendaciones}

Los resultados arrojados por la presente investigación, realizada con estudiantes de $6^{\circ}$ a $11^{\circ}$ grado de la Institución Educativa San Juan Bosco de Palermo, permiten realizar sugerencias sobre otros estudios que se pueden desarrollar en la institución y que a su 
vez facilitarían el comprender el fenómeno de la sexualidad y dar respuesta a nuevos interrogantes. Por lo tanto existe la necesidad de realizar investigaciones sociales para la implementación de estrategias que se enfoquen al cambio de actitudes y prácticas erróneas tanto en hombres y mujeres en lo referente a la educación sexual y dar respuesta a nuevos interrogantes. Uno de ellos corresponde a estudios como:

Conocer cuál es la percepción de los padres de familia y profesores frente a prácticas, conocimientos y opiniones que poseen sobre la sexualidad identificando si en ellos persisten posturas enfocadas a cada una de las variables de estudio, cuya perspectiva ubica en el proceso de desarrollo sexual como una educación, conocimiento, información y manejo de estrategias de orientación, toma de decisiones informadas, saludables, responsabilidad y valores en los adolescentes, o posturas que guardan relación con las creencias populares que se transmiten culturalmente. Así mismo, se plantea una segunda línea de investigación acerca de las estrategias I.E.C (Informar, Educar, Comunicar) planeadas a través del Plan de Intervenciones Colectivas de impacto en la población adolescente, con el objetivo de determinar el cumplimiento de la norma en este tipo de población por parte del sector gobierno y la cobertura del servicio en el Municipio de Palermo.

Desde la línea de la psicología se plantean otras recomendaciones que conciernen a aquellas acciones enfocadas a la prevención o intervención de esta problemática y se establecería: Desarrollar estrategias de prevención sexual en los estudiantes, teniendo en cuenta tres ejes sobre los cuales deben desarrollarse: 1. La adecuada información sobre las prácticas sexuales, 2. La instrucción y educación sobre los conocimientos sexuales 3 . Las adecuadas opiniones sobre la sexualidad para proyectar una adecuada comunicación con lenguaje adecuado al tema.

\section{Referencias Bibliográficas}

Pandi, Agencia Periodismo Amigo de los Derechos de la Infancia (2006). Tomado el 19 de Agosto de 2013: Aumenta explotación y abuso sexual de niños en Colombia y se encuentra disponible en http://www.semana.com/on-line/articulo/aumenta-explotacionabuso-sexual-ninos-colombia/86676-3. Revista Semana, sección La Nación, 2007)

OEA"Comisión Interamericana de Derechos Humanos (CDH)" Informe Anual 2011 (Informe de gestión para la garantía de los derechos de la infancia, adolescencia y juventud - Dpto. del Huila, Neiva Julio 2011.

Informes sobre VIH/SIDA, departamento del Huila, 1988-2012, observados en el Boletín epidemiológico departamento del Huila, (2012).

Secretaria de Educación del Huila, área de Cobertura Educativa-grupo de acceso matricula oficial 2013.SIMAT Anexo \&A 01-06-2013.

Briones, G. (1996). "Metodología de la investigación cuantitativa en las ciencias sociales". www. monografías.com. Recuperado el 20 de noviembre de 2007.

Ortiz Sáenz Ana L. (2010) (página 10) Actividad sexual en el adolescente. Fundación Labor y Amor. Bogotá.

Ortiz S., Ana Levis, (2010). Importante orientación familiar en adolescentes. Ponencia Internacional en Educación Género y Sexo. Medellín. (Página 7).

Ortiz S., Ana Levis. 2011. La Práctica Sexual en adolescentes y jóvenes una realidad. Fundación Labor y Amor. Bogotá, (Página 22-25-28).

Cubillos, José $O$. Orientación a Parejas de adolescentes y jóvenes. Sagrados Corazones Belgas, Bogotá 2011 . (Página 44, 45).

Caricote Agreda, Esther A. Influencia de los padres en la educación sexual de los Adolescentes. Educere, Meridad, v. 12, n. 40, marzo 2008. pp. 79-87. Disponible en

http://www.scielo.org.ve/scielo.php?script=sci_arttext\&pid=\$13 16-49102008000100010\&ing=es\&nrm=iso.

Cubillos R. Elvia (2010), (Página 39) Sexo y Erotismo ¿un buen conocimiento? Bogotá.

Plan local de salud reproductiva del municipio de Palermo- Huila, Secretaria de Salud Municipio de Palermo, 2008-2011. Disponible en dirección electrónica: http://www.palermo-huila.gov.co/apcaa-files/39656238386334363436373961343033/PLAN_LOCAL_ DE_SSR_4.pdf

Mojica, D. (2011). El goce de la sexualidad y sus deformaciones. Página 67-87. Editorial Graflip. Bogotá.

Franco Vega Rosalba noviembre 2010. "Situación de las madres solteras, visto desde la teoría de Erving Goffman". Página 14,23. (Observado 26 de julio en:

http://www.uaeh.edu.mx/nuestro_alumnado/esc_sup/actopan/ licenciatura/Situacion\%20de\%20las\%20madres\%20solteras.pdf

Carrasco, R. (2007, 16 de enero). La sexualidad en el adolescente: Conformación de la conciencia en el adolescente sobre la sexualidad. Revista PsicologiaCientifica.com, 9(3). (Página 2 del artículo) Disponible en:

http://www.psicologiacientifica.com/sexualidad-adolescente

Babatunde Osotimehin, Director ejecutivo de UNFPA. Educación integral sobre la sexualidad apropiada. 10 - VII-2013. Boletín de la ONU, Comunicado No. 13/11510 de julio 2013 (Única hoja). Disponible en: http://www.cinu.mx/comunicados/2013/07/ mensaje-del-director-ejecutivo-1/. 